\title{
CHARACTERISTICS OF RF-SPUTTERED COCr FILMS
}

T. Wielinga, J.C. Lodder and J. Worst

\section{ABSTRACT}

The magnetization of the $\mathrm{CoCr}$ recording medium has been investigated by several methods. First the perpendicular hysteresis loops are analysed in the thickness range from 500 to $20,000 \AA$. This provided evidence that the magnetization process is typefied by domain wall motion. Second the dependence of the coercivity on the film thickness has been determined. The dependence found can be explained if it is assumed, that the coercivity is caused by domain walls, impeded by the crystallite boundaries. Finally stand-still recording experiments have been performed, which confirm that magnetization takes place by the displacement of domain walls. The switching criterion in the writing process is best met by taking the field averaged over the film thickness.

\section{INTRODUCTION}

Under optimized conditions, RF-sputtering has been shown to be a useful method for the deposition of $\mathrm{CoCr}$ layers for perpendicular recording (1). It has also been shown, that films with excellent c-axis orientation, which is practically independent of the film thickness $h$, are obtained if silicon (100) or mica/carbon substrates are used (2). By means of torque measurements it has been demonstrated, that the magnetic anisotropy, due to this excellent c-axis orientation of the entire film volume, is also practically independent of the $f i l m$ thickness (3). The second order anisotropy constant $\mathrm{K}_{2}$ of the uniaxial anisotropy appeared to be only a few percent of the first order anisotropy constant $\mathrm{K}_{1}(3)$, and therefore the anisotropy is sufficient1y described by $K_{1}$ only. The saturation magnetization $M_{S}$, measured by means of a VSM, has also been shown to be independent of the film thickness, due to the constant chemical composition (3). The crystallite diameter appeared to increase with the film thickness (2), resulting in a film morphology, which is marked by conical crystallites (see Fig. 1). Table I presents the relevant magnetic properties of the two $\mathrm{CoCr}$ compositions examined.

\begin{tabular}{|l|l|l|}
\hline $\mathrm{at} \% \mathrm{Cr}$ & 16 & 19 \\
\hline $\mathrm{M}_{\mathrm{s}}\left(\mathrm{emu} / \mathrm{cm}^{3}\right)$ & 680 & 450 \\
$\mathrm{~K}_{1}\left(\mathrm{erg} / \mathrm{cm}^{3}\right)$ & $22 \times 10^{5}$ & $9 \times 10^{5}$ \\
$\mathrm{~K}_{2}\left(\mathrm{erg} / \mathrm{cm}^{3}\right)$ & $0.8 \times 10^{5}$ & $0.2 \times 10^{5}$ \\
$\mathrm{H}_{\mathrm{k}}(\mathrm{k} 0 \mathrm{e})$ & 7.0 & 3.0 \\
$\Delta \theta_{50}^{*}\left({ }^{\circ}\right)$ & 1.5 & 2.0 \\
$\mathrm{~S}^{*}$ & 0.04 & 0.05 \\
\hline
\end{tabular}

*for 1 um thick films

Table I. The magnetic properties of 16 and 19 at\% $\mathrm{Cr}$ films.

The magnetic micro-structure of CoCr films is commonly considered to consist of single-domain particles, coinciding with the crystallites, and therefore rotation as the main switching mechanism (4). In contrast with this single domain particle model, we found experimental evidence that the magnetization mechanism has clear features of domain wall motion. The perpendicular hysteresis loops follow the behaviour as predicted by Kooy and Enz (5) for a stripe domain structure and using

Manuscript received June 8, 1982.

Department of Electrical Engineering, Twente University of Technology, 7500 AE Enschede, The Netherlands.

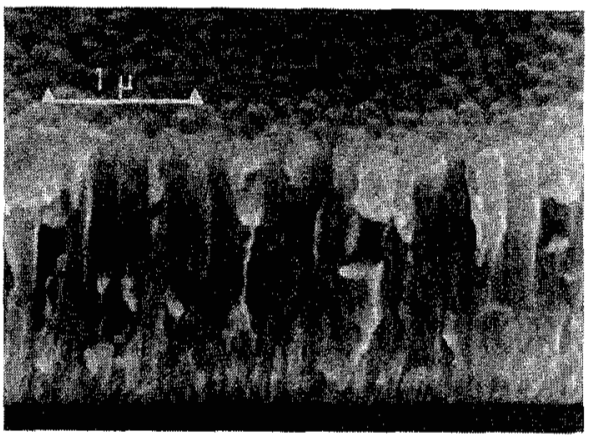

Fig. 1. SEM photograph of the fractional structure of a 2 micron thick CoCr layer, showing conical crystalIites.

a method, which is based on this model (6), the Bloch domain wall energy $\sigma_{w}$ could be determined.

The perpendicular coercivity field $\mathrm{H}_{\mathrm{c}}$ has been measured as a function of the film thickness, or even better, the surface crystallite diameter. The results also indicate a magnetization reversal mechanism by domain walls, hindered by the crystallite boundaries. This type of coercivity explains the maximum of $\mathrm{H}_{c}$ found at a certain $f i l m$ thickness or crystallite diameter.

By means of stand-still recording experiments $(1,10)$, the switching criterion has been determined, i.e. where the switching in the film can be localized. It appears that the switching is governed by the total field (i.e. the head field and the demagnetizing field), averaged over the film thickness. This is in agreement with a switching mechanism by vertical Bloch walls.

\section{THE PERPENDICULAR HYSTERESIS LOOP}

The magnetization reversal has been primarily investigated by studying the perpendicular hysteresis loops of the CoCr recording medium, measured by means of a VSM. Some features of these loops indicate a magnetization process, characterized by domain wall motion (see Fig. 2). The decrease of $M$ from saturation shows a "shoulder" which is typical for stripe domain behaviour and further $D$, the slope of the hysteresis loop at $M=0$ $\left(=4 \pi(\mathrm{dM} / \mathrm{dH})_{\mathrm{M}=0}\right)$, appears to be strongly dependent on the film thickness (see Fig. 5).

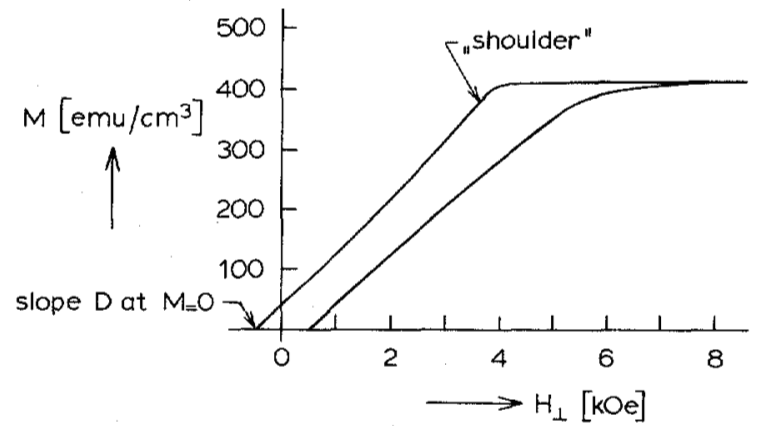

Fig. 2. The perpendicular hysteresis loop of a 19 at\% Cr film ( 1.14 micron thick), exhibiting some features of domain wall switching.

The hysteresis loops of magnetic films with perpendicular anisotropy and vertical Bloch walls, were described first by Kooy and Enz (5). Their results have been applied by Dekker (6) in the case of MnBi films. We used his method of determining the Bloch wall energy $\sigma_{W}$ from the slope $D$ at $M=0$ of the perpendicular hysteresis loop. Figure 3 shows the model of the domain configuration for which the calculations are carried out. 


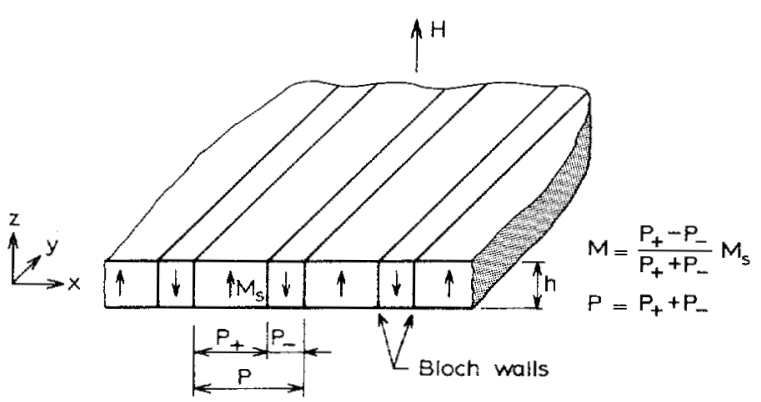

Fig. 3. The stripe domain configuration with Bloch walls, used for the calculation of $M$ and $P$.

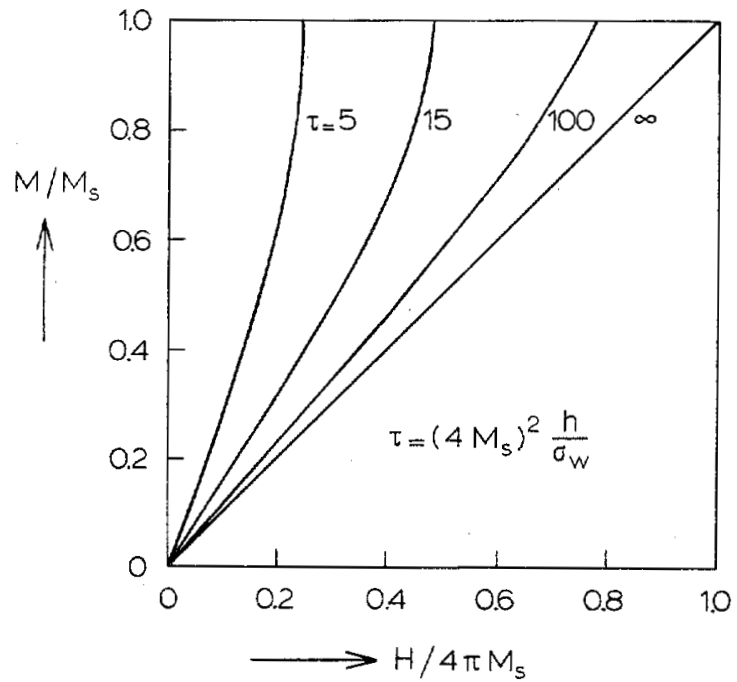

Fig. 4. Some calculated, perpendicular magnetization curves for several values of the reduced film thickness $\tau=\left(4 \mathrm{M}_{\mathrm{s}}\right)^{2} \mathrm{~h} / \mathrm{\sigma}_{\mathrm{w}}(5)$.

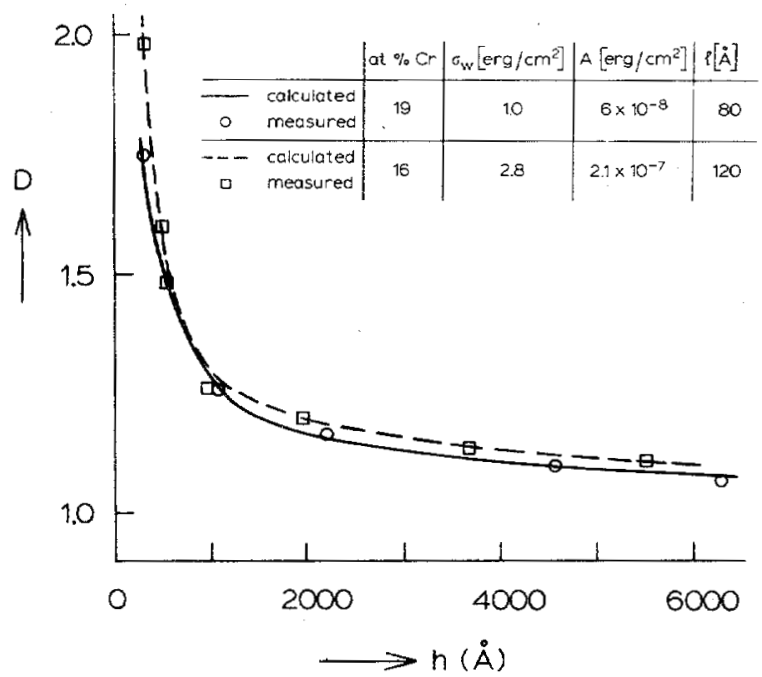

Fig. 5. The hysteresis loop slope $D$ at $M=0$ as a function of the film thickness $h$ for the two different $\mathrm{CoCr}$ compositions used.

The magnetization $M$ and the domain period $P$ can be calculated from the equations (9) and (10), as derived by Kooy and Enz. In Fig. 4 some calculated magnetization curves are shown for several values of the reduced thickness $\tau$, assuming zero coercivity. Clearly the slope $D$ at $H=0$ depends strongly on $h$ and can therefore be used to determine $\sigma_{W}$. Taking $M=0$ and the derivative of their eq. (9), the slope $D$ and the domain period $P$ at $M=0$ are obtained from:

$$
\begin{aligned}
& \mathrm{D}^{-1}=1+\frac{2 \sqrt{\mu}}{1+\sqrt{\mu}} \frac{1}{\pi \alpha_{0}} \ln \left\{\frac{1+\exp \left(-2 \pi \alpha_{0}\right)}{2}\right\} \\
& \frac{\sigma_{W}}{\left(4 M_{s}\right)_{h}^{2}} \frac{1+\sqrt{\mu}}{2 \sqrt{\mu}}=\frac{1}{4 \pi^{2} \alpha_{0}^{2}} \sum_{n=1}^{\infty} \frac{1}{(2 n+1)^{3}} \times \\
& \times\left[1-\left\{1+2 \pi(2 n+1) \alpha_{0}\right\} \exp \left\{-2 \pi(2 n+1) \alpha_{0}\right\}\right]
\end{aligned}
$$

Herein is $\mu=1+2 \pi \mathrm{M}_{S}^{2} / \mathrm{K}_{1}, \underline{\alpha}_{0}=\mathrm{h} \sqrt{\mu} / \mathrm{P}_{0}$ and $\sigma_{\mathrm{w}}$ the Bloch wall energy: $\sigma_{\mathrm{w}}=4 \sqrt{\mathrm{AK}_{1}}$ where $\mathrm{A}$ is the exchange constant. For the Cocr films used, $\mu$ is about 2.2 , so that this $\mu$-correction is quite significant. The measured hysteresis loops differ from the calculated ones by their coercivity. Because this merely causes a shift of the magnetization curve along the $\mathrm{H}$-axis if $M$ is not too large, we can take $\mathrm{D}$ at $\mathrm{H}=\mathrm{H}_{\mathrm{c}}$ in the experimental loops. It is assumed that $\sigma_{W}$ is independent of the film thickness. This is justified by the fact that $K_{1}$ and $M_{S}$ (and therefore also A) are practically independent of the film thickness. Furthermore, because the critical film thickness (7) for Bloch walls in CoCr filns is about $400 \AA$, only Bloch walls are assumed. Figure 5 presents the measured slope $D$ against the film thickness together with the calculated $D$ according to eq. (1) and (2), adjusting $\sigma_{w}$ to give the best fit. A good agreement is obtained. If $\sigma_{w}$ is known, the exchange constant $A$ and the Bloch wall thickness $Z=\pi \sqrt{A} / K_{1}$ can be calculated (see inset of Fig. 5). In Figure 6 the measured loop is compared with curves, calculated according to the free energy model of Kooy and Enz and the restricted free energy model, as proposed by Dekker. Using the first model, the curve, denoting the magnetization process from the state of saturation, can be described. In the latter it is assumed, that the domain walls are pinned to strong pinning centers and therefore in the approach to saturation the domain period remains constant $\mathrm{P}_{\mathrm{O}}$. In this model M saturates at $\left(\mathrm{H}-\mathrm{H}_{\mathrm{C}}\right)=4 \pi \mathrm{M}_{\mathrm{S}}$.

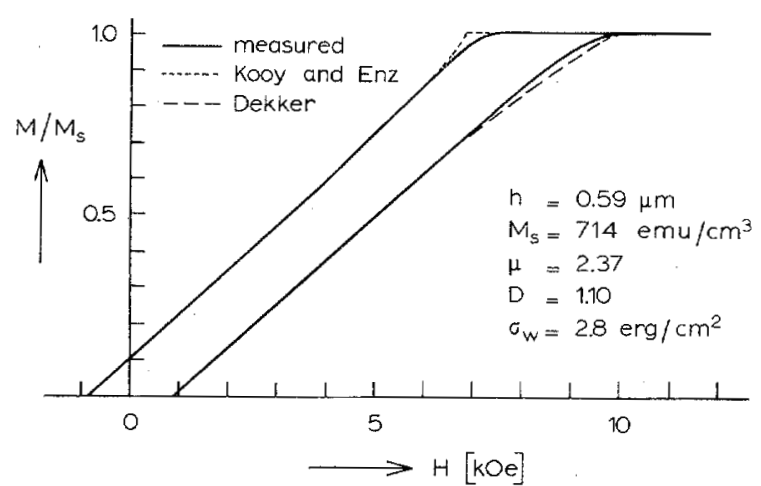

Fig. 6. Measured and calculated hysteresis curves according to (5) and (6).

\section{COERCIVITY}

For the two CoCr compositions used, $\mathrm{H}_{\mathrm{C}}$ has been measured as a function of the film thickness (see fig. 7. Both exhibit a maximum but at different film thicknesses or crystallite diameters (the latter is denoted above the horizontal axis of Fig. 7). This can be explained, if it is assumed that the domain walls are impeded by the crystallite boundaries, having a local minimum wall energy. Thus there exists a periodical change of the wall energy caused by these crystallite boundaries. For this type of domain wall coercivity, it has been shown that the coercitive field exhibits a maximum if $z$ is of the same order of magnitude as $d(8)$. Because the dependence of $\mathrm{d}$ on $\mathrm{h}$ is the same for both compositions, the shift of their maxima can be explained by their different wall thicknesses $?$. 
Moreover, the maximum value of $\mathrm{H}_{c}$ is about the same for both compositions, which have greatly differing anisotropy fields (see Table I). This contradicts the supposition, that the coercivity is related to rotational magnetization.

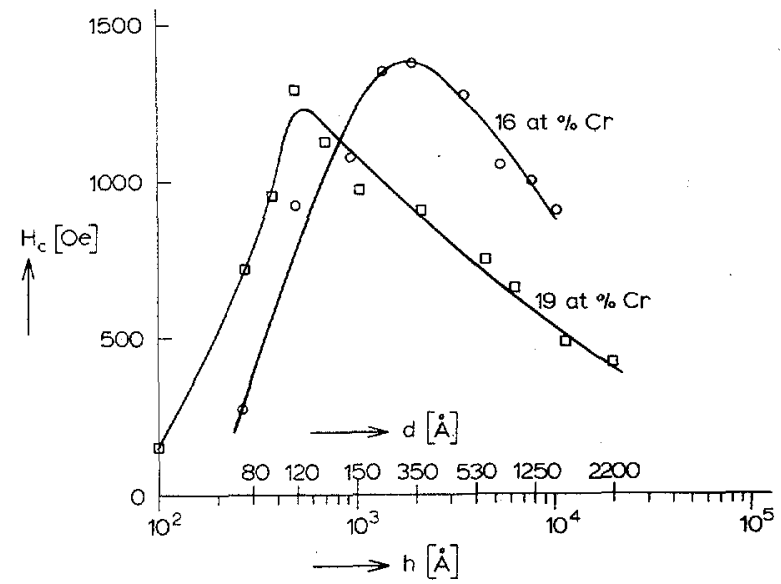

Fig. 7. The perpendicular coercitive field $\mathrm{H}_{c}$, plotted against the film thickness $h$ and crystallite diameter d of 16 and 19 at\% Cr films.

\section{STAND-STILL RECORDING EXPERIMENTS}

The resultant local magnetization in the recording medium during writing is determined by the head field and the demagnetizing field in relation to the medium coercivity. Due to the relative high anisotropy field and the smallin-plane remanence of the $\mathrm{CoCr}$ medium, the recording process is sufficiently described by an one dimensional model, taking into account only vertical magnetization $(1,9)$. This model is restricted to standstill recording. We used a Permalloy single pole type (SPT) head for writing and a magneto-resistive transducer (MRT) for reading. Figure 8 shows a typical MRT response of a SPT head print in a $\mathrm{CoCr}$ medium.

In the calculations according to this one-dimensional model, a choice has to be made about where the switching can be located in the medium, i.e. where the switching criterion is met. In the first instance this problem can be considered as mathematical.

To detect the switching location, the following procedure was followed: a) the 4.5 micron thick SPT head is activated at several distances $s$ from the 2 micron thick CoCr medium, b) the induced head prints are read out by the MRT and c) the measured MRT response is compared with computer simulated MRT responses, calculated by taking the total field $\mathrm{H}$ at different switching locations in the recording medium namely at the top $\left(\mathrm{H}_{\mathrm{z}=+\mathrm{h} / 2}\right)$, bottom $\left(\mathrm{H}_{\mathrm{z}=-\mathrm{h} / 2}\right)$ and middle $\left(\mathrm{H}_{\mathrm{z}=0}\right)$ and also taking the fields, averaged over the film thickness (H).

\section{$\mathrm{S}=0.50 \mu \mathrm{m}$}

MRT to medium distance $=0.75 \mu \mathrm{m}$

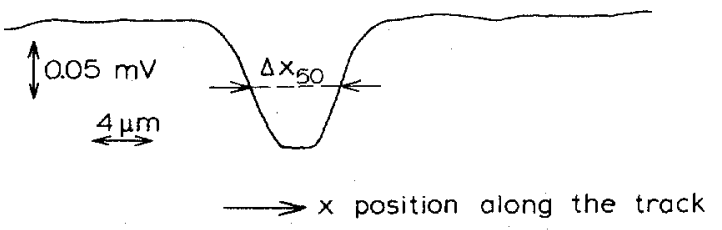

Fig. 8. A typical MRT response of a stand-still SPT head print in a CoCr layer; $\Delta \mathrm{X}_{50}$ is the half-output voltage width.

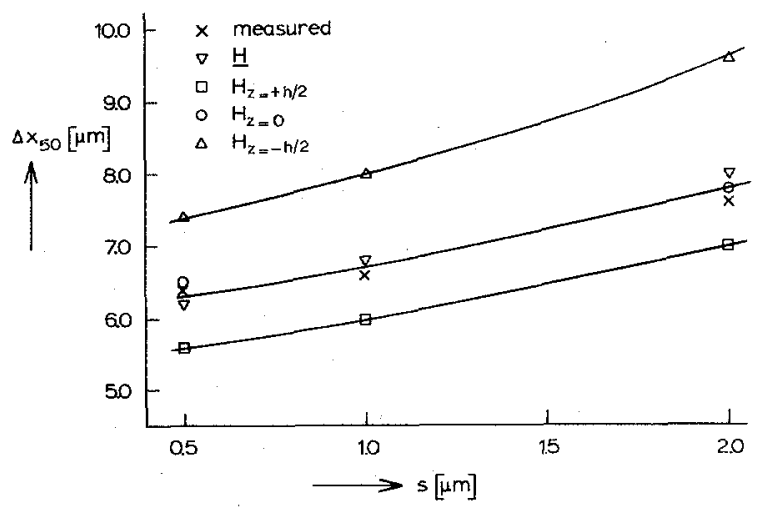

Fig. 9. The half-output voltage width $\Delta \mathrm{x}_{50}$ for several head-medium distances $\mathrm{s}$; measured as well as calculated for different switching locations in the recording medium.

For the comparison of the different MRT responses, we have taken the half-output vol tage width $\Delta \mathrm{X}_{50}$ of the MRT response (see Fig. 8). An analytical expression is taken for the SPT head field in the calculations (10). Figure 9 shows the results of the experimental as well as the calculated $\Delta \mathrm{X}_{50}$ for several head medium distances s. Clearly the average field and the field at the middle of the film give the best agreement with experiments.

To give physical interpretation of this result, we can say that it is consistent with a magnetization through domain wall motion because its displacement is governed by the average field across the wall.

\section{ACKNOWLEDGEMENTS}

We are grateful to J.H.J. Fluitman and G.H. Jonker for their stimulating and helpful discussions and to all the students who have contributed to this work.

\section{REFERENCES}

1. T. Wielinga, J.C. Lodder, IEEE Trans. Magn., Vol. MAG-17, No. 6, 1981.

2. J.C. Lodder, T. Wielinga and J. Worst, submitted to "Thin Solid Films".

3. J. Worst, J.C. Lodder and T. Wielinga, submitted to "Thin Solid Films".

4. G. Iwasaki, K. Ouchi and N. Honda, IEEE Trans. Magn., Vol. MAG-16, No. 5, 1980.

5. C. Kooy and U. Enz, Philips Res. Repts 15, 1960.

6. P. Dekker, Thesis 1974, Delft University of Technology, Delft, The Netherlands.

7. J.C. Hendy, H.D. Richards and A.W. Simpson, J. of Mat. Sc. 1, 1966.

8. S. Chikazumi, Physics of Magnetism, New York: Wiley, 1964, pp. 206-207.

9. T. Wielinga, J.H.J. Fluitman and J.C. Lodder, submitted to IEEE Trans. Magn.

10. T.J. Szczech, M. Steinback and M. Jodeit, IEEE Trans. Magn., Vol. MAG-18, No. 1, 1982. 